eine Lösung von $13,1 \mathrm{~g}$ (0,06 Mol) Methyl-cyclopentadienyl-mangan-tricarbonyl und 5,9 g (0,075 Mol) Acetylchlorid in $60 \mathrm{~cm}^{3}$ Schwefelkohlenstoff zugetropft. Unter HCl-Entwicklung scheidet sich dabei eine dunkelrote Zwischenverbindung ab. Nach Beendigung des $\mathrm{Zu}$ tropfens wird noch 45 Min. unter Rühren auf dem Wasserbad zum Sieden erhitzt. Zu dem abgekühlten Reaktionsgemisch werden nach dem Abdekantieren des Schwefelkohlenstoffs unter Rühren 100 \% Eis und $10 \mathrm{~cm}^{3}$ konz. Salzsäure gegeben. Das abgeschiedene dunkle Öl wird im Scheidetrichter mit $250 \mathrm{~cm}^{3}$ Benzol und die wäßrige Lösung nochmals mit $150 \mathrm{~cm}^{3}$ Benzol ausgeschüttelt. Die vereinigten Benzolauszüge werden nach gründlichem Waschen mit Wasser und nach dem Trocknen mit $\mathrm{CaCl}_{2}$ i. Vak. bis zum zurückbleibenden dunkelroten Öl $(15 \mathrm{~g})$ eingeengt. Das Öl wird dann i. Vak. destilliert, es siedet zwischen 111 und $113^{\circ} / 0,7$ Torr. Die Ausbeute beträgt $13 \mathrm{~g}(83,2 \% \mathrm{~d}$. Th.) eines roten Öls.

$\mathrm{C}_{11} \mathrm{H}_{9} \mathrm{O}_{4} \mathrm{Mn}(260,11) \quad$ Ber. $\quad$ C 50,79 H 3,49 .

In entsprechender Weise konnten bei der Umsetzung mit Benzoylchlorid erhalten werden:

$\mathrm{C}_{16} \mathrm{H}_{11} \mathrm{O}_{4} \mathrm{Mn} \quad(322,18)$

a) gelbe Nadeln, Schmp. $121^{\circ}$;

b) gelbe Nadeln, Schmp. $58^{\circ}$;

Ausbeute a) $47 \%$, b) $32 \%$.

I-Äthylierung :

In einem 250- $\mathrm{cm}^{3}$-Dreihalskolben, versehen mit Rückflußkühler, KPG-Rührer und Tropftrichter, werden zu
$35 \mathrm{~g} \quad(0,26 \mathrm{Mol})$ Aluminiumchlorid $50 \mathrm{~g}$ Äthylbromid gegeben. Unter Rühren und Erhitzen auf einem Wasserbad von $40^{\circ}$ Badtemperatur wird ein Gemisch von $40 \mathrm{~g}$ $(0,18 \mathrm{Mol}) \mathrm{I}$ und $100 \mathrm{~g}$ Äthylbromid innerhalb von 2,5 Stdn. zugetropft. Die Reaktion läuft unter starker Halogenwasserstoff-Entwicklung ab. Es wird dann noch 3 Stdn. unter weiterem Rühren zum Sieden erhitzt und anschließend das überschüssige Äthylbromid abdestilliert. Der dunkelrote Rückstand wird nach dem Abkühlen unter Rühren mit Eiswasser zersetzt, das abgesetzte dunkle Öl abgetrennt und die wäßrige Phase mit etwa $400 \mathrm{~cm}^{3}$ Benzol extrahiert. Benzollösung und Öl werden mehrmals mit verd. Salzsäure und anschließend bis zur neutralen Reaktion mit Wasser gewaschen. Nach dem Trocknen mit $\mathrm{CaCl}_{2}$ wird das Lösungsmittel unter Stickstoff i. Vak. abgezogen und das zurückbleibende dunkle Öl (44,5 g) unter Stickstoff im Hochvakuum fraktioniert destilliert.

Es konnten hierbei neben anderen Fraktionen folgende Äthyl-I-Derivate isoliert werden:

Mono-äthyl-I, Sdp. $71,5-72^{\circ}$ bei 0,15 Torr, $n_{\mathrm{D}}{ }^{20}: 1,5708$. $\mathrm{C}_{11} \mathrm{H}_{11} \mathrm{O}_{3} \mathrm{Mn}(246,13) \quad$ Ber. C 53,67 H 4,50 .

Gef. C 53,30 H 4,70 .

Di-äthyl-I, Sdp. $98,5-100,5^{\circ}$ bei 0,3 Torr, $n_{\mathrm{D}}{ }^{20}: 1,5571$.

$\mathrm{C}_{13} \mathrm{H}_{15} \mathrm{O}_{3} \mathrm{Mn}(274,18) \quad$ Ber. C 56,94 H 5,51 .

Gef. C 57,30 H 5,70 .

Tri-äthyl-I, Sdp. $114-116^{\circ}$ bei 0,25 Torr, $n_{\mathrm{D}}{ }^{20}: 1,5527$. $\mathrm{C}_{15} \mathrm{H}_{19} \mathrm{O}_{3} \mathrm{Mn}(302,23) \quad$ Ber. C 59,61 H 6,34 .

Gef. C 60,10 H 6,30 .

Wir danken Herrn Dr. W. B. Ligetr, USA., für die Uberlassung wert vollen Aisgangsmaterials.

\section{Wechselwirkung tertiärer aromatischer Phosphine mit Alkalimetallen}

Von K. IssLeib und H. O. Fröhlich

Anorganisch-chemisches Institut der Friedrich-SchillerUniversität Jena

(Z. Naturforschg. 14 b, 349-350 [1959] ; eingegangen am 28. Februar 1959)

In Fortführung der Versuche über die Metallketylbildung aus Alkaliphosphor-Verbindungen - u. a. auch $\left(\mathrm{C}_{6} \mathrm{H}_{5}\right)_{2} \mathrm{PLi}$ - mit aromatischen Ketonen ${ }^{1}$ haben wir für die Darstellung der Alkaliphosphor-Verbindungen unabhängig von Wittenberg und Gilman ${ }^{2}$ die Wechselwirkung tertiärer aromatischer Phosphine mit Lithium, Natrium und Kalium untersucht. Für die Umsetzung schied Tetrahydrofuran als Lösungsmittel aus, da es uns nicht gelang, unter diesen Bedingungen die Diphenylphosphinalkalimetall-Verbindungen $-\mathrm{R}_{2} \mathrm{PMe}-$ rein $\mathrm{zu}$ isolieren, was auch $\mathrm{W}_{\text {ItTenberg }}$ und Gilman ${ }^{2}$ bestätigten. Einfach und leicht zugänglich waren Diphenyl-

1 K. Issleib u. A. Tzschach, Naturwissenschaften 44, 452 [1957].

2 G. Wittenberg u. H. Gilman, J. org. Chem. 23, 1063 [1958]. Diphenylphosphonlithium wurde von den Autoren nicht in Substanz isoliert. phosphinnatrium und -kalium, als Triphenylphosphin und Natrium bzw. Kalium in absolutem Dioxan unter $\mathrm{N}_{2}$ erhitzt wurden.

Das $\left(\mathrm{C}_{6} \mathrm{H}_{5}\right)_{2} \mathrm{PK} \cdot 2$ Dioxan wurde nach Einengen der filtrierten dunkelroten Reaktionslösung in Form orangeroter Kristalle in einer Ausbeute von 70\% der Theorie erhalten. Nach Zugabe von Wasser zu der Reaktionslösung und anschließender Destillation wurde das $\mathrm{Di}$ phenylphosphin in gleicher Ausbeute [bez. auf $\left(\mathrm{C}_{6} \mathrm{H}_{5}\right)_{3} \mathrm{P}$ ] rasch zugänglich.

Das Diphenylphosphinnatrium ${ }^{3}$ wurde auf die gleiche Weise dargestellt. Die Ausbeute betrug nur 25\% der Theorie, obwohl $\left(\mathrm{C}_{6} \mathrm{H}_{5}\right)_{3} \mathrm{P}$ und $\mathrm{Na}$ im Molverhältnis $1: 3,5$ eingesetzt und längere Zeit (16 Stdn.) in Dioxan gekocht wurden. Das gelbe $\left(\mathrm{C}_{6} \mathrm{H}_{5}\right)_{2} \mathrm{PNa}$ kristallisiert mit 1,3 Molen Dioxan.

Die entsprechende Lithiumverbindung ${ }^{4}$ konnte nach diesem Verfahren nicht isoliert werden, da die Reaktionslösung zu viel Nebenprodukte infolge Reaktion des Phenyllithiums mit Dioxan enthielt. Das $\left(\mathrm{C}_{6} \mathrm{H}_{5}\right)_{2} \mathrm{PLi}^{5}$

3 W. Kuchen u. H. Buchwald, Angew. Chem. 69, 307 [1957], erhielten $\left(\mathrm{C}_{6} \mathrm{H}_{5}\right)_{2} \mathrm{PNa}$ aus $\left(\mathrm{C}_{6} \mathrm{H}_{5}\right)_{2} \mathrm{PCl}$ und $\mathrm{Na}$.

4 W. Rüdorff u. W. Müller, Dissertation W. Müller, Tübingen 1957, spalteten $\left(\mathrm{C}_{6} \mathrm{H}_{5}\right)_{3} \mathrm{P}$ mit $\mathrm{Li}$ in flüssigem $\mathrm{NH}_{3} \mathrm{zu}$ $\left(\mathrm{C}_{6} \mathrm{H}_{5}\right)_{3} \mathrm{PLi}$.

5 K. Issleib u. A. Tzschach, Chem. Ber., im Druck. 
wurde daher aus $\left(\mathrm{C}_{6} \mathrm{H}_{5}\right){ }_{2} \mathrm{PH}$ und $\mathrm{C}_{6} \mathrm{H}_{5} \mathrm{Li}$ rein dargestellt.

Das bei der Umsetzung des Triphenylphosphins mit Kalium gemäß der Gleichung

$$
\left(\mathrm{C}_{6} \mathrm{H}_{5}\right)_{3} \mathrm{P}+2 \mathrm{~K} \rightarrow \mathrm{KP}\left(\mathrm{C}_{6} \mathrm{H}_{5}\right)_{2}+\mathrm{KC}_{6} \mathrm{H}_{5}
$$

entstandene Phenylkalium setzte sich bei den hohen Reaktionstemperaturen mit Dioxan vollständig um; denn Einleiten von $\mathrm{CO}_{2}$ in die Reaktionslösung lieferte keine Benzoesäure. Ganz analoge Beobachtungen trafen für die Reaktion des $\left(\mathrm{C}_{6} \mathrm{H}_{5}\right)_{3} \mathrm{P}$ mit $\mathrm{Na}$ zu. Wurde dagegen $\left(\mathrm{C}_{6} \mathrm{H}_{5}\right)_{3} \mathrm{P}$ mit einer Kalium-Natrium-Legierung $(4: 1)$ in Dioxan bei Zimmertemperatur und unter Außenkühlung des Reaktionsgefäßes gerührt und gleichzeitig $\mathrm{CO}_{2}$ eingeleitet, so konnte nach Hydrolyse Benzoesäure ohne weiteres isoliert werden.

Wir nehmen an, daß bei der Wechselwirkung des $\left(\mathrm{C}_{6} \mathrm{H}_{5}\right)_{3} \mathrm{P}$ mit Alkalimetallen diese zunächst addiert werden und im weiteren Verlauf der Reaktion das intermediär gebildete TriphenylphosphindialkalimetallAddukt in Diphenylphosphinalkalimetall und Alkalimetallphenyl zerfällt. Diese Vermutung wird dadurch gestützt, daß das wesentlich mesomeriefähigere Trixenylphosphin in Dioxan 2 Atome Kalium bzw. Natrium addiert. Aus der filtrierten, intensiv bordeauxroten Lösung des Reaktionsproduktes von $\left(\mathrm{C}_{6} \mathrm{H}_{5} \mathrm{C}_{6} \mathrm{H}_{4}\right)_{3} \mathrm{P}$ und Kalium wurde nach Einengen und Zugabe von Äther ein rotes Reaktionsprodukt erhalten, aus dessen Analysenergebnissen sich ein Atomverhältnis von $\mathrm{P}: \mathrm{K}=1: 2,2$ errechnete ${ }^{6}$. Im Gegensatz zum Triphenylphosphin führt die Einwirkung von Kalium auf Trixenylphosphin (bei $50-60^{\circ} \mathrm{C}$ ) nicht zur Abspaltung eines Xenylrestes, denn nach Hydrolyse und Oxydation war keine Dixenylphosphinsäure nachweisbar. Die Umsetzung des $\left(\mathrm{C}_{6} \mathrm{H}_{5} \mathrm{C}_{6} \mathrm{H}_{4}\right)_{3} \mathrm{P}$ mit $\mathrm{Na}$ verlief analog. In Tetrahydrofuran erfolgte die Addition der Alkalimetalle an das Trixenylphosphin schon bei Zimmertemperatur; über Einzelheiten hierzu wird später berichtet.

${ }^{6}$ Die zu hohen Kaliumwerte deuten auf geringfügige Hydrolyse hin.

\section{Vinyllead Compounds}

\section{By Bodo Bartocha and M. Y. Gray}

Chemistry Division, U.S. Naval Ordnance Laboratory, Corona, California U.S.A.

(Z. Naturforschg. 14 b, 350—351 [1959] ; eingegangen am 2. März 1959)

Tetravinylblei wurde durch Behandlung von Kaliumhexachloroplumbat mit einem Uberschuß von Vinylmagnesiumbromid hergestellt. Zur Darstel lung der gemischten Vinyl- $\mathrm{A}$ thyl-Bleiverbindungen, $\mathrm{Pb}\left(\mathrm{C}_{2} \mathrm{H}_{5}\right)_{\mathrm{n}}\left(\mathrm{C}_{2} \mathrm{H}_{3}\right)_{4-\mathrm{n}}$, verwendet man die Chloride oder Bromide $\mathrm{Pb}\left(\mathrm{C}_{2} \mathrm{H}_{5}\right){ }_{n} \mathrm{X}_{4-\mathrm{n}}$ und läßt darauf eine Vinyl-Grign a rd-Verbindung einwirken.

The discovery of the vinyl $\mathrm{G} \mathrm{rig} \mathrm{n}$ a r d reagent ${ }^{1}$ has permitted the preparation of vinyl compounds of several metals and non-metals ${ }^{2}$. Current interest in vinyl-deri-

1 H. Normant, C. R. hebd. Séances Acad. Sci. 239, 1510 [1954].

2 B. Bartocha and F. G. A. Stone, Z. Naturforschg. 13 b, 347
Weitere Versuche ergaben, daß das Tri- $\alpha$-naphthyl. phosphin beispielsweise mit Kalium gespalten wird. Es sind Untersuchungen im Gange, Verbindungen des Typs $\mathrm{R}^{\prime} \mathrm{R}_{2} \mathrm{P}$ und $\mathrm{R}^{\prime} \mathrm{R}^{\prime \prime} \mathrm{R}^{\prime \prime} \mathrm{P}$ mit Alkalimetallen umzusetzen, um die unterschiedliche Abspaltung der verschiedenen Reste zu studieren.

Triäthyl- und Tricyclohexylphosphin, als Vertreter aliphatischer und cycloaliphatischer Phosphine, reagierten auch bei Variation der Reaktionsbedingungen nicht mit Lithium, Natrium und Kalium.

Die Alkyl-, Cycloalkyl- und Arylderivate der Alkaliphosphor-Verbindungen ${ }^{5}$ des Typs $\mathrm{MePR}_{2}$ und $\mathrm{Me}_{2} \mathrm{PR}$ sind besonders luft- und feuchtigkeitsempfindlich. Diese Verbindungsklasse zeichnet sich durch große Reaktionsfreudigkeit aus. So wurden, um nur einige Beispiele anzuführen, aus $\mathrm{BrCH}_{2}-\mathrm{CH}_{2} \mathrm{Br}$ und $\left(\mathrm{C}_{6} \mathrm{H}_{11}\right)_{2} \mathrm{PLi}$ das $\left(\mathrm{C}_{6} \mathrm{H}_{11}\right)_{2} \mathrm{P}-\mathrm{CH}_{2} \mathrm{CH}_{2}-\mathrm{P}\left(\mathrm{C}_{6} \mathrm{H}_{11}\right)_{2}$, Schmp. 167 bis $169^{\circ} \mathrm{C}$, aus $\mathrm{CH}_{3} \mathrm{COCl}$ und $\left(\mathrm{C}_{6} \mathrm{H}_{5}\right)_{2} \mathrm{PNa}$ das $\mathrm{CH}_{3} \mathrm{C}(\mathrm{O}) \mathrm{P}\left(\mathrm{C}_{6} \mathrm{H}_{5}\right)_{2}$, Sdp. $143-146^{\circ} \mathrm{C}(2,5 \mathrm{~mm} \mathrm{Hg})$, aus $\left(\mathrm{C}_{6} \mathrm{H}_{5}\right)_{3} \mathrm{PO}$ und $\left(\mathrm{C}_{6} \mathrm{H}_{5}\right)_{2} \mathrm{PLi}$ das rote $\left(\mathrm{C}_{6} \mathrm{H}_{5}\right)_{3} \mathrm{PO}$ - $\mathrm{LiP}\left(\mathrm{C}_{6} \mathrm{H}_{5}\right)_{2}$ und aus $\mathrm{ClCH}_{2} \mathrm{C}(\mathrm{O}) \mathrm{OC}_{2} \mathrm{H}_{5}$ und $\left(\mathrm{C}_{6} \mathrm{H}_{5}\right)_{2} \mathrm{PNa}$ der Phosphinosäureester $\left(\mathrm{C}_{6} \mathrm{H}_{5}\right)_{2} \mathrm{P}$ - $\mathrm{CH}_{2} \mathrm{C}(\mathrm{O}) \mathrm{OC}_{2} \mathrm{H}_{5}$, Sdp. $186-187^{\circ} \mathrm{C}(5 \mathrm{~mm} \mathrm{Hg})$ bzw. nach Verseifen die Säure $\left(\mathrm{C}_{6} \mathrm{H}_{5}\right)_{2} \mathrm{P}-\mathrm{CH}_{2}-\mathrm{COOH}$, Schmp. $117-118^{\circ} \mathrm{C}$, erhalten.

Über die Darstellung der Alkaliphosphor-Verbindungen $\mathrm{R}_{2} \mathrm{PMe}$ und $\mathrm{RPMe}_{2}$ sowie deren Umsetzungen mit Dihalogenalkanen, anorganischen und organischen Säurechloriden, Halogencarbonsäureestern, Ketonen, Aldehyden, Nitrosoverbindungen, Phosphinoxyden, Nitrilen, $\mathrm{C}-\mathrm{C}-, \mathrm{N}-\mathrm{N}$ - bzw. CN-Doppelbindungs-Systemen, Äthylenoxyd, Äthylenimin, Metallhalogeniden, $\mathrm{NO}, \mathrm{CO}_{2}$ und $\mathrm{SO}_{2}$ wird in den Chem. Ber. unter dem Titel „Alkali-Phosphorverbindungen und ihr reaktives Verhalten" ausführlich berichtet. Außerdem wird das komplexchemische Verhalten der zuvor erwähnten phosphororganischen Verbindungen gegenüber Metallsalzen untersucht und an anderer Stelle mitgeteilt.

Herrn Prof. Dr. Dr. h. c. Fr. Hein danken wir für die fördernde Unterstützung dieser Arbeiten.

vatives of metals has prompted us to report two new vinyllead compounds which we are using to prepare vinyl compounds of group III and V elements by utilizing a method similar to one previously described ${ }^{3}$. A more detailed account of this work will be published in the near future.

This communication deals with the preparation of tetravinyllead and divinyldiethyllead. We have found that tetravinyllead can be made in good yield by the addition of potassium hexachloroplumbate, $\mathrm{K}_{2} \mathrm{PbCl}_{6}$, to a solution of vinylmagnesium bromide or chloride in tetrahydrofuran. The new compound has the general properties of alkyl-lead compounds; it is stable to air, water and light and can be stored at room temperature for months. Divinyldiethyllead was obtained in yields up

[1958] and earlier work cited therein.

3 B. Bartocha, F. E. Brinckman, H. D. Kaesz and F. G. A. Stone, Proc. chem. Soc. 1958, 116. 